(1988) [61-68]

\title{
ELEMENTARY EQUTVALENCE AND GENUS OF FINITELY GENERATED NILPOTENT GROUPS
}

Francis Oger

\begin{abstract}
We show that, if two finitely generated nilpotent groups are elementarily equivalent, and more generally if they satisfy the same sentences with one alternation of quantifiers, then, they are quite similar, though not necessarily isomorphic. For instance, each of them is isomorphic to a subgroup of finite index of the other and they have the same finite images, the same Mistin genus and the same Pickel genus.
\end{abstract}

\section{DEFinitions aND MAIN RESUlt}

The notion of nilpotent group is defined, for instance, in [1] and [6]. We say that a group $G$ is finite-by-abelian (respectively finite-by-nilpotent) if there exists a finite normal subgroup $H$ of $G$ such that $G / H$ is abelian (respectively nilpotent).

The reader can find in $[6$, Theorem 8.5, p. 63] the definition of the $\pi$-localisation $G_{\pi}$ of a nilpotent group $G$ for a set $\pi$ of prime numbers. We denote by $G_{0}$ and $G_{p}$ respectively the $\pi$-localisation of $G$ for $\pi=\emptyset$ and $\pi=\{p\}$, where $p$ is a prime number.

If $S$ is a subset of a group $G$, we denote by $\langle S\rangle$ the subgroup of $G$ which is generated by $S$.

The notions of formula, sentence (or formula without free variable) and elementary equivalence are defined in [2]. A formula with one alternation of quantifiers is written $\varphi=\left(\exists x_{1} \ldots \exists x_{n}\right)\left(\forall y_{1} \ldots \forall y_{p}\right) \theta$ or $\varphi=\left(\forall x_{1} \ldots \forall x_{n}\right)\left(\exists y_{1} \ldots \exists y_{p}\right) \theta$ with $\theta$ a formula without quantifiers.

The following "similarity criteria" are often considered for two finitely generated nilpotent groups $G, H$ :

a) Commensurability $[1$, p. 9]: $G$ and $H$ are commensurable if they contain subgroups of finite index $\tilde{G}, \tilde{H}$ with $\tilde{G} \cong \tilde{H}$;

b) There exist injective homomorphisms from $G$ to $H$ and from $H$ to $G$;

c) $G$ and $H$ have the same finite images if, for each finite group $F$, the existence of a surjective homomorphism from $G$ to $F$ is equivalent to the existence of a surjective homomorphism from $H$ to $F$;

d) Pickel Genus [7, p. 125]: $G$ and $H$ have the same Pickel genus if they have the same finite images and satisfy $G_{o} \cong H_{o}$;

Received 4 March 1987

Copyright Clearance Centre, Inc. Serial-fee code: 0004-9729/88 \$A2.00+0.00. 
e) Mislin Genus [7, p. 125]: $G$ and $H$ have the same Mislin genus if they satisfy $G_{p} \cong H_{p}$ for each prime number $p$;

f) $G$ and $H$ satisfy $G_{\pi} \cong H_{\pi}$ for each finite set $\pi$ of prime numbers;

g) For each integer $n \geqslant 1$, there exists an integer $d$ which is prime to $n$, a subgroup $\tilde{G}$ of $G$ such that $G^{d} \subseteq \tilde{G}$ and $\tilde{G} \cong H$, and a subgroup $\tilde{H}$ of $H$ such that $H^{d} \subseteq \tilde{H}$ and $\tilde{H} \cong G$.

Each of the properties $a), b), c), d$ ), e) and $f$ ) is a consequence of $g$ ). The implications $\mathbf{g}) \Rightarrow \mathrm{b}) \Rightarrow \mathrm{a}$ ) and $\mathbf{f}) \Rightarrow \mathrm{e}) \Rightarrow \mathrm{d}) \Rightarrow \mathrm{c}$ ) are easily proved.

In order to prove $g) \Rightarrow f$ ), Lemma 8.7 of $[6]$ allows us to restrict ourselves to sets $\pi$ which contain the prime divisors of the cardinal of the torsion subgroup of $G$. If $\pi$ is such a set, the canonical homomorphism from $G$ to $G_{\pi}$ is injective by Theorem 8.9 of [6]. According to g), there exists an integer $d$ which is prime to the elements of $\pi$ and a subgroup $\bar{G}$ of $G$ such that $G^{d} \subseteq \tilde{G}$ and $\tilde{G} \cong H$. For each $x \in G_{\pi}$, there exists an integer $k$ which is prime to the elements of $\pi$ and such that $x^{k} \in G$; we have $x^{k d} \in \bar{G}$ and $k d$ is prime to the elements of $\pi$. This implies $G_{\pi}=\tilde{G}_{\pi}$, whence $G_{\pi} \cong H_{\pi}$ since $\tilde{G}$ is isomorphic to $H$.

This proof remains valid if we replace $\mathrm{g}$ ) by the following property:

$\left.\mathrm{g}^{\prime}\right)$ For each integer $n \geqslant 1$, there exists an integer $d$ which is prime to $n$ and a subgroup $\bar{G}$ of $G$ such that $G^{d} \subseteq \tilde{G}$ and $\tilde{G} \cong H$.

It follows from the Theorem below that two finitely generated nilpotent groups $G$, $H$ satisfy a), b), c), d), e), f), g) if they are elementarily equivalent.

Two finitely generated nilpotent groups can be elementarily equivalent without being isomorphic, even if they are finite-by-abelian ([4, Corollary 5.6]) or torsion-free ([5, Section 2]).

THEOREM. If $G$ and $H$ are finitely generated finite-by-nilpotent groups which satisfy the same sentences with one alternation of quantifiers, then, for each integer $m \geqslant 1$, there exists an integer $d$ which is prime to $m$, a subgroup $\tilde{G}$ of $G$ such that $G^{d} \subseteq \tilde{G}$ and $\tilde{G} \cong H$, and a subgroup $\tilde{H}$ of $H$ such that $H^{d} \subseteq \tilde{H}$ and $\tilde{H} \cong G$.

The following "similarity criterion" has been considered, in particular, by Hirshon and Warfield (see [3] and [7]):

h) $G \times \mathbf{Z} \cong H \times \mathbf{Z}$.

It implies elementary equivalence for any groups $G, H$, according to [5].

In the case of finitely generated finite-by-abelian groups, elementary equivalence is equivalent to both the properties $\mathrm{c}$ ), h) by [4, Theorem 5.5, p. 478].

So far, we have not obtained such characterisations of elementary equivalence for finitely generated finite-by-nilpotent groups. In particular, we do not know whether, for such groups, elementary equivalence is equivalent to $h$ ). 
The following question also seems open: Is the problem of knowing whether two finite presentations of finite-by-nilpotent groups define elementarily equivalent groups recursively soluble?

\section{Proof of The Theorem}

As the hypotheses concerning $G$ and $H$ are symmetrical, it suffices to prove that, for each integer $m \geqslant 1$, there exists an integer $d$ which is prime to $m$ and an injective homomorphism $f: G \rightarrow H$ such that $H^{d} \subseteq f(G)$.

The set $t(G)$ of all torsion elements of $G$ is a finite normal subgroup of $G$ and $G / t(G)$ is a torsion-free nilpotent group. We define the subgroups $T_{i}(G)$ by $T_{o}(G)=$ $t(G)$ and, for each integer $i \geqslant 1, T_{i}(G)=\left\{x \in G \mid\right.$ for each $\left.y \in G,[x, y] \in T_{i-1}(G)\right\}$. For each integer $i \geqslant 1$, we have $T_{i}(G) / t(G)=Z_{i}(G / t(G))$, where $\left(Z_{j}(G / t(G))\right)_{j \in N^{*}}$ is the upper central series of $G / t(G)$; so, $Q_{i}(G)=T_{i}(G) / T_{i-1}(G)$ is abelian and torsion-free.

We define similarly $t(H)$ and, for each integer $i, T_{i}(H)$ and $Q_{i}(H)$. These groups satisfy the properties stated above for $t(G)$ and the subgroups $T_{i}(G)$ and $Q_{i}(G)$.

We consider an integer $q \geqslant 1$ such that $t(G)^{q}=\{1\}$ and $t(H)^{q}=\{1\}$; we define universal formulae without parameters $\theta_{i}(x)$ by $\theta_{o}(x)=$ " $x^{q}=1$ " and, for each integer $i \geqslant 1, \theta_{i}(x)="(\forall y) \theta_{i-1}([x, y]) "$. For each integer $i$, the formula $\theta_{i}(x)$ characterises $T_{i}(G)$ in $G$ and $T_{i}(H)$ in $H$.

We denote by $s$ the smallest integer $i$ such that the universal sentence $(\forall x) \theta_{i}(x)$ is true in $G$ (or, equivalently, in $H$ ). The groups $G / t(G)$ and $H / t(H)$ are nilpotent of class $s$ and $s$ is the smallest integer $i$ such that $T_{i}(G)=G$ (or, equivalently, $\left.T_{i}(H)=H\right)$.

We denote by $u_{1}, \ldots, u_{r}$ the elements of $t(G)$ and we consider a family $\left(u_{i, j}\right)_{1 \leqslant i \leqslant 0,1 \leqslant j \leqslant t(i)}$ of elements of $G$ such that, for each $i \in\{1, \ldots, s\}$, the images of $u_{i, 1}, \ldots, u_{i, t(i)}$ modulo $T_{i-1}(G)$ form a basis of the $Z$-module $T_{i}(G) / T_{i-1}(G)$. Each element of $G$ can be written in a unique way $x=u_{a} \prod_{i=1}^{*}\left(\prod_{j=1}^{t(i)} u_{i, j}^{a(i, j)}\right)$ with $1 \leqslant a \leqslant r$ and $a(i, j) \in \mathbf{Z}$ for $1 \leqslant i \leqslant s$ and $1 \leqslant j \leqslant t(i)$. Moreover, the structure of $G$ is completely determined by the relations:

$$
\begin{aligned}
& u_{a} u_{b}=u_{k(a, b)} \text { for } 1 \leqslant a \leqslant r \text { and } 1 \leqslant b \leqslant r \\
& {\left[u_{a}, u_{b, c}\right]=u_{k(a, b, c)} \text { for } 1 \leqslant a \leqslant r, 1 \leqslant b \leqslant s \text { and } 1 \leqslant c \leqslant t(b)} \\
& {\left[u_{a, b}, u_{c, d}\right]=u_{k(a, b, c, d)} \prod_{i=1}^{s}\left(\Pi_{j=1}^{t(i)} u_{i, j}^{k(i, j, a, b, c, d)}\right) \text { for } 1 \leqslant a \leqslant s, 1 \leqslant} \\
& b \leqslant t(a), 1 \leqslant c \leqslant s \text { and } 1 \leqslant d \leqslant t(c) \text { with } k(i, j, a, b, c, d)=0 \text { for } \\
& i \geqslant \inf (a, c) .
\end{aligned}
$$


We denote by $R_{G}\left(x_{1}, \ldots, x_{r},\left(x_{i, j}\right)_{1 \leqslant i \leqslant 0,1 \leqslant j \leqslant t(i)}\right)$ the formula

$$
\begin{aligned}
& \left(\bigwedge_{1 \leqslant a \leqslant r}^{1 \leqslant b \leqslant r} x_{a} x_{b}=x_{k(a, b)}\right) \wedge\left(\bigwedge_{1 \leqslant a \leqslant r}\left(\bigwedge_{1 \leqslant b \leqslant 0}^{1 \leqslant c \leqslant t(b)}\left[x_{a}, x_{b, c}\right]=x_{k(a, b, c)}\right)\right) \\
& \wedge\left(\bigwedge_{1 \leqslant a \leqslant 0}^{1 \leqslant b \leqslant t(a)}\left(\bigwedge_{1 \leqslant c \leqslant s}^{1 \leqslant d \leqslant t(c)}\left[x_{a, b}, x_{c, d}\right]=x_{k(a, b, c, d)} \prod_{i=1}^{s}\left(\prod_{j=1}^{t(i)} x_{i, j}^{k(i, j, a, b, c, d)}\right)\right)\right) .
\end{aligned}
$$

By Proposition 2.1 of [4], for each integer $n$, there exists an integer $k(n)$ such that each element of $G^{n}$ can be written $y_{1}^{n} \ldots y_{k(n)}^{n}$ with $y_{1}, \ldots, y_{k(n)} \in G$ and each element of $H^{n}$ can be written $y_{1}^{n} \ldots y_{k(n)}^{n}$ with $y_{1}, \ldots, y_{k(n)} \in H$. We denote by $\psi_{n}(y)$ the existential formula without parameters $\left(\exists y_{1} \ldots \exists y_{k(n)}\right)\left(y=y_{1}^{n} \ldots y_{k(n)}^{n}\right)$, which characterises $G^{n}$ in $G$ and $H^{n}$ in $H$.

For each integer $n \geqslant 2$, we denote by $E(n)$ the (finite) set which consists of the elements $\left(a,(a(i, j))_{1 \leqslant i \leqslant \bullet, 1 \leqslant j \leqslant t(i)}\right)$, with $1 \leqslant a \leqslant r$ and $0 \leqslant a(i, j) \leqslant n-1$ for $1 \leqslant i \leqslant s$ and $1 \leqslant j \leqslant t(i)$, such that $u_{a} \prod_{i=1}^{s}\left(\prod_{j=1}^{t(i)} u_{i, j}^{a(i, j)}\right)$ does not belong to $G^{n}$. We consider the sentence:

$$
\emptyset_{n}=\left(\exists x_{1} \ldots \exists x_{r} \exists\left(x_{i, j}\right)_{1 \leqslant i \leqslant s, 1 \leqslant j \leqslant t(i)}\right) \varphi_{n}\left(x_{1}, \ldots, x_{r},\left(x_{i, j}\right)_{1 \leqslant i \leqslant 0,1 \leqslant j \leqslant t(i)}\right)
$$

where $\varphi_{n}$ is the formula

$$
\begin{aligned}
R_{G}\left(x_{1}, \ldots, x_{r},\left(x_{i, j}\right)_{1 \leqslant i \leqslant s, 1 \leqslant j \leqslant t(i)}\right) & \wedge\left(\bigwedge_{1 \leqslant i \leqslant s}^{1 \leqslant j \leqslant t(i)} \theta_{i}\left(x_{i, j}\right)\right) \\
& \wedge\left(\bigwedge_{E(n)} \neg \psi_{n}\left(x_{a} \prod_{i=1}^{s}\left(\prod_{j=1}^{t(i)} x_{i, j}^{a(i, j)}\right)\right)\right) .
\end{aligned}
$$

The formula $\varphi_{n}$ is equivalent to a universal formula since it is a conjunction of universal formulas. So, the sentence $\emptyset_{n}$ is equivalent to a $\exists \forall$ sentence.

For each integer $n \geqslant 2$, as the sentence $\theta_{n}$ is true in $G$, it is also true in $H$, and the formula $\varphi_{n}$ is satisfied by a sequence $v_{1}, \ldots, v_{r},\left(v_{i, j}\right)_{1 \leqslant i \leqslant s, 1 \leqslant j \leqslant t(i)}$ of elements of $H$; we define an homomorphism $f_{n}: G \rightarrow H$ by writing $f_{n}\left(u_{h}\right)=v_{h}$ for $1 \leqslant h \leqslant r$ and $f_{n}\left(u_{i, j}\right)=v_{i, j}$ for $1 \leqslant i \leqslant s$ and $1 \leqslant j \leqslant t(i)$.

We have $f_{n}\left(T_{o}(G)\right) \subseteq T_{o}(H)$ since $f_{n}\left(T_{o}(G)\right)$ is a finite subgroup of $H$. We also have $f_{n}\left(T_{i}(G)\right) \subseteq T_{i}(H)$ for each integer $i \geqslant 1$ because, for each integer $i$ such that $1 \leqslant i \leqslant s$, the formula $\theta_{i}$ is satisfied by each of the elements $v_{i, 1}, \ldots, v_{i, t(i)}$. 
The homomorphism $f_{n}$ induces an injective homomorphism from $G / G^{n}$ to $H / H^{n}$. In fact, we have $f_{n}\left(G^{n}\right)=\left(f_{n}(G)\right)^{n} \subseteq H^{n}$ and we prove that $f_{n}^{-1}\left(H^{n}\right)$ is contained in $G^{n}$ as follows:

For each sequence $\left(a,(a(i, j))_{1 \leqslant i \leqslant s, 1 \leqslant j \leqslant t(i)}\right)$, with $1 \leqslant a \leqslant r$ and $a(i, j) \in Z$ for $1 \leqslant i \leqslant s$ and $1 \leqslant j \leqslant t(i)$, we consider the sequence $\left(c,(c(i, j))_{1 \leqslant i \leqslant s, 1 \leqslant j \leqslant t(i)}\right)$ defined by the conditions $c=a, c(i, j)-a(i, j) \in n Z$ and $0 \leqslant c(i, j) \leqslant n-1$ for $1 \leqslant i \leqslant s$ and $1 \leqslant j \leqslant t(i)$. The elements

$$
\begin{aligned}
& \left(u_{a} \prod_{i=1}^{s}\left(\prod_{j=1}^{t(i)} u_{i, j}^{a(i, j)}\right)\right)^{-1}\left(u_{c} \prod_{i=1}^{s}\left(\prod_{j=1}^{t(i)} u_{i, j}^{c(i, j)}\right)\right) \quad \text { and } \\
& \left(v_{a} \prod_{i=1}^{s}\left(\prod_{j=1}^{t(i)} v_{i, j}^{a(i, j)}\right)\right)^{-1}\left(v_{c} \prod_{i=1}^{\Delta}\left(\prod_{j=1}^{t(i)} v_{i, j}^{c(i, j)}\right)\right)
\end{aligned}
$$

respectively belong to $G^{n}$ and $H^{n}$ since $G^{n}$ (respectively $H^{n}$ ) is a normal subgroup of $G$ (respectively $H$ ) which contains the $n$-th powers of the elements of $G$ (respectively $H)$.

If $f_{n}\left(u_{a} \prod_{i=1}^{s}\left(\prod_{j=1}^{t(i)} u_{i, j}^{a(i, j)}\right)\right)=v_{a} \prod_{i=1}^{s}\left(\prod_{j=1}^{t(i)} v_{i, j}^{a(i, j)}\right)$ belongs to $H^{n}$, then, the same property is true for $v_{c} \prod_{i=1}^{s}\left(\prod_{j=1}^{t(i)} v_{i, j}^{c(i, j)}\right)$ and $\left(c,(c(i, j))_{1 \leqslant i \leqslant s, 1 \leqslant j \leqslant t(i)}\right)$ does not belong to $E(n)$ since the sequence $v_{1}, \ldots, v_{r},\left(v_{i, j}\right)_{1 \leqslant i \leqslant s, 1 \leqslant j \leqslant t(i)}$ satisfies the formula $\varphi_{n}$ in $H$. So, $u_{c} \prod_{i=1}^{s}\left(\prod_{j=1}^{t(i)} u_{i, j}^{c(i, j)}\right)$ belongs to $G^{n}$ and the same property is true for $u_{a} \prod_{i=1}^{s}\left(\prod_{j=1}^{t(i)} u_{i, j}^{a(i, j)}\right)$.

For each integer $i$ such that $1 \leqslant i \leqslant s$, the restriction of $f_{n}$ to $T_{i}(G)$ is an homomorphism from $T_{i}(G)$ to $T_{i}(H)$ which induces an injective homomorphism from $T_{i}(G) /\left(T_{i}(G) \cap G^{n}\right)$ to $T_{i}(H) /\left(T_{i}(H) \cap H^{n}\right)$. It follows that $\left|T_{i}(G) /\left(T_{i}(G) \cap G^{n}\right)\right| \leqslant$ $\left|T_{i}(H) /\left(T_{i}(H) \cap H^{n}\right)\right|$, whence, since the hypotheses concerning $G$ and $H$ are symmetrical, $\left|T_{i}(G) /\left(T_{i}(G) \cap G^{n}\right)\right|=\left|T_{i}(H) /\left(T_{i}(H) \cap H^{n}\right)\right|$. So, the restriction of $f_{n}$ to $T_{i}(G)$ induces an isomorphism from $T_{i}(G) /\left(T_{i}(G) \cap G^{n}\right)$ to $T_{i}(H) /\left(T_{i}(H) \cap H^{n}\right)$. In particular, $f_{n}$ induces an isomorphism from $G / G^{n}$ to $H / H^{n}$.

Consequently, for each integer $i$ such that $1 \leqslant i \leqslant s$, the restriction of $f_{n}$ to $T_{i}(G)$ induces a surjective homomorphism from $T_{i}(G) /\left\langle T_{i-1}(G), T_{i}(G) \cap G^{n}\right\rangle$ to $T_{i}(H) /\left\langle T_{i-1}(H), T_{i}(H) \cap H^{n}\right\rangle$. It follows that $\left|T_{i}(G) /\left\langle T_{i-1}(G), T_{i}(G) \cap G^{n}\right\rangle\right| \geqslant$ $\left|T_{i}(H) /\left\langle T_{i-1}(H), T_{i}(H) \cap H^{n}\right\rangle\right|$, whence, since the hypotheses concerning $G$ and $H$ are symmetrical, $\left|T_{i}(G) /\left\langle T_{i-1}(G), T_{i}(G) \cap G^{n}\right\rangle\right|=\left|T_{i}(H) /\left\langle T_{i-1}(H), T_{i}(H) \cap H^{n}\right\rangle\right|$. So, the restriction of $f_{n}$ to $T_{i}(G)$ induces an isomorphism from $T_{i}(G) /\left\langle T_{i-1}(G), T_{i}(G) \cap G^{n}\right\rangle$ to $T_{i}(H) /\left\langle T_{i-1}(H), T_{i}(H) \cap H^{n}\right\rangle$. 
By Proposition 2.5 of [4], for each integer $m \geqslant 2$, there exists an integer $n \geqslant 2$ such that each element of $H^{n}$ is the $m$-th power of an element of $H$. We associate with $n$ the sequence $u_{1}, \ldots, u_{r},\left(u_{i, j}\right)_{1 \leqslant i \leqslant 0,1 \leqslant j \leqslant t(i)}$ of elements of $G$, the sequence $v_{1}, \ldots, v_{r},\left(v_{i, j}\right)_{1 \leqslant i \leqslant s, 1 \leqslant j \leqslant t(i)}$ of elements of $H$, the sentence $\emptyset_{n}$ and the homomorphism $f_{n}$ defined above.

For each integer $i$ such that $1 \leqslant i \leqslant s, T_{i}(H)$ is generated by $T_{i-1}(H), T_{i}(H) \cap H^{n}$ and $f_{n}\left(T_{i}(G)\right)$. It follows from the definition of $n$ that any element $y \in T_{i}(H) \cap H^{n}$ is the $m$-th power of an element $z$ which necessarily belongs to $T_{i}(H)$ like $y$ since $H / T_{i}(H)$ is torsion-free. So, $T_{i}(H)$ is generated by $T_{i-1}(H), T_{i}(H)^{m}$ and $f_{n}\left(T_{i}(G)\right)$. The torsion-free abelian group $Q_{i}(H)=T_{i}(H) / T_{i-1}(H)$ is therefore generated by $Q_{i}(H)^{m}$ and the images $z_{i, 1}, \ldots, z_{i, t(i)}$ of the elements $v_{i, 1}, \ldots, v_{i, t(i)}$ modulo $T_{i-1}(H)$.

This implies that there exists an integer $d$ which is prime to $m$ and such that $Q_{i}(H)^{d}$ is contained in $\left\langle z_{i, 1}, \ldots, z_{i, t(i)}\right\rangle$.

In fact, for any basis $\left\{w_{1}, \ldots, w_{t}\right\}$ of the free $\mathrm{Z}$-module $Q_{i}(H)$, we have

$$
\begin{aligned}
& w_{1}=w_{1}^{m a(1,1)} \quad \ldots \quad w_{t}^{m a(1, t)} x_{1} \\
& w_{t}=w_{1}^{m a(t, 1)} \quad \ldots \quad w_{t}^{m a(t, t)} x_{t}
\end{aligned}
$$

with $x_{1}, \ldots, x_{t} \in\left\langle z_{i, 1}, \ldots, z_{i, t(i)}\right\rangle$ and $a(i, j) \in Z$ for $1 \leqslant i, j \leqslant s$. It follows from the equalities

$$
\begin{aligned}
& x_{1}=w_{1}^{1-m a(1,1)} w_{2}^{-m a(1,2)} \quad \ldots \quad w_{t}^{-m a(1, t)} \\
& x_{2}=w_{1}^{-m a(2,1)} w_{2}^{1-m a(2,2)} \quad \ldots \quad w_{t}^{-m a(2, t)} \\
& x_{t}=w_{1}^{-m a(t, 1)} w_{2}^{-m a(t, 2)} \quad \ldots \quad w_{t}^{1-m a(t, t)}
\end{aligned}
$$

that the subgroup of $Q_{i}(H)$ which is generated by $x_{1}, \ldots, x_{t}$ contains $w_{1}^{d}, \ldots, w_{t}^{d}$ for

$$
d=\mid \begin{array}{cccc}
1-m a(1,1) & -m a(1,2) & \vdots & -m a(1, t) \\
-m a(2,1) & 1-m a(2,2) & \vdots & -m a(2, t) \\
\ldots \ldots \ldots \ldots \ldots \ldots \ldots \ldots \ldots \ldots \ldots \ldots \ldots \ldots \ldots \ldots & \ldots \ldots \ldots
\end{array} \ldots .
$$

So, the rank of the free $\mathrm{Z}$-module $Q_{i}(H)$ is at most equal to the rank $t(i)$ of the free $\mathrm{Z}$-module $Q_{i}(G)=T_{i}(G) / T_{i-1}(G)$. As a matter of fact, as the hypotheses concerning $G$ and $H$ are symmetrical, $Q_{i}(G)$ and $Q_{i}(H)$ have same rank.

Therefore, $z_{i, 1}, \ldots, z_{i, t(i)}$ form a free subset of $Q_{i}(H)$ and the restriction of $f_{n}$ to $T_{i}(G)$ induces an injective homomorphism from $Q_{i}(G)$ to $Q_{i}(H)$. As this property is 
true for each integer $i$ such that $1 \leqslant i \leqslant s$ and the restriction of $f_{n}$ to $T_{o}(G)$ is an isomorphism from $T_{o}(G)$ to $T_{o}(H)$, it follows that $f_{n}$ is an injective homomorphism.

We can suppose for the remainder of the proof that $m \geqslant 2$ and that $m$ is divisible by all prime numbers $p \leqslant s$, because the theorem is true for each integer $m \geqslant 1$ if we show that it is true for each integer $m \geqslant 2$ which is divisible by $s !$.

According to the result that we proved above, there exists an integer $d$ which is prime to $m$ and such that $T_{i}(H)^{d}$ is contained in $\left\langle f_{n}\left(T_{i}(G)\right), T_{i-1}(H)\right\rangle$ for each integer $i$ such that $1 \leqslant i \leqslant s$.

The integer $d$ has no prime divisor $p \leqslant s$, in view of our hypothesis concerning $m$. It is easy to show, by adapting the proof of Corollary 2.31 of [1], that, in the nilpotent group $H / T_{o}(H)$, for each integer $i \geqslant 1$, each product of $d^{i}$-th powers is a $d^{i}$-th power. So, any element of $H^{d^{i}}$ can be written $x=y^{d^{i}} z$ with $y \in H$ and $z \in T_{o}(H)$.

We show by induction on the integer $i \leqslant s$ that $T_{i}(H)^{d^{i}}$ is contained in $f_{n}\left(T_{i}(G)\right)$. This result is true for $i=0$ since the restriction of $f_{n}$ to $T_{o}(G)$ is an isomorphism from $T_{o}(G)$ to $T_{o}(H)$. So, we can suppose $i \geqslant 1$.

According to the definition of $d$, for each $x \in T_{i}(H)$, there exists an element $y \in f_{n}\left(T_{i}(G)\right)$ such that $x^{d} \in y T_{i-1}(H)$. We have $x^{d^{i}} \in y^{d^{i-1}} T_{i-1}(H)$ since $T_{i-1}(H)$ is normal in $H$. The element $\left(y^{d^{i-1}}\right)^{-1} x^{d^{i}} \in T_{i-1}(H) \cap H^{d^{i-1}}$ can be written $z^{d^{i-1}} w$ with $z \in H$ and $w \in T_{o}(H)$. We have $z^{d^{i-1}} \in T_{i-1}(H)$ since $w$ and $z^{d^{i-1}} w$ belong to $T_{i-1}(H)$. As $H / T_{i-1}(H)$ is torsion-free, $z$ also belongs to $T_{i-1}(H)$. So, $z^{d^{i-1}}$ belongs to $T_{i-1}(H)^{d^{i-1}}$ and therefore to $f_{n}\left(T_{i-1}(G)\right)$ by the induction assumption. Consequently, $x^{d^{i}}=y^{d^{i-1}} z^{d^{i-1}} w$ belongs to $f_{n}\left(T_{i}(G)\right)$ since $y^{d^{i-1}}, z^{d^{i-1}}$ and $w$ respectively belong to $f_{n}\left(T_{i}(G)\right), f_{n}\left(T_{i-1}(G)\right)$ and $T_{o}(H)=f_{n}\left(T_{o}(G)\right)$.

It follows that $H^{d^{*}}$ is contained in $f_{n}(G)$.

\section{REFERENCES}

[1] G. Baumslag, Lecture Notes on Nilpotent Groups, (Regional Conference Series in Math. 2, Amer. Matl. Soc., Providence, Rhode Island 1971).

[2] C.C. Chang and H.J. Keisler, Model Theory, (Studies in Logic 73, North-Holland, Amsterdam 1973).

[3] R. Hirshon, 'The equivalence of $\times{ }^{t} C \approx \times{ }^{t} D$ and $J \times C \approx J \times D$ ', Trans. Amer. Math. Soc. $24 \theta$ (1979), 331-340.

[4] F. Oger, 'Equivalence élémentaire entre groupes finis-par-abéliens de type fini', Comment. Math. Helv. 57 (1982), 469-480.

[5] F. Oger, 'Cancellation and elementary equivalence of groups', J. Pure Appl. Alg. 30 (1983), 293-299.

[6] R.B. Warfield, Jr., Nilpotent Groups, (Lecture Notes in Math. 513, Springer-Verlag, Berlin 1976).

[7] R.B. Warfield, Jr., 'Genus and cancellation for groups with finite commutator subgroup', J. Pure Appl. Alg. 6 (1975), 125-132. 
Département de Mathématiques Université Paris VII

2 place Jussieu

75.251 Paris Cedex 05, France
12 rue de la Gare,

89.410 Cézy,

France. 